\title{
Analysis of Plant Origin Antibiotics against Oral Bacterial Infections Using In Vitro and In Silico Techniques and Characterization of Active Constituents
}

\author{
Abdul Rafey ${ }^{1}$, Adnan Amin ${ }^{1}$, Muhammad Kamran ${ }^{1}$, Uzma Haroon ${ }^{2}$, Kainat Farooq ${ }^{3}$, Kenn Foubert ${ }^{4}$ \\ and Luc Pieters $4, *$ (D) \\ 1 NPRL, Gomal Centre of Pharmaceutical Sciences, Faculty of Pharmacy, Gomal University, \\ Dera Ismail Khan 29050, Pakistan; abdulrafe4667@gmail.com (A.R.); \\ adnan.amin@gu.edu.pk (A.A.); m.kamran.gu@gmail.com (M.K.) \\ 2 Department of Dentistry, D.H.Q Teaching Hospital, Dera Ismail Khan 29050, Pakistan; \\ uzma.haroonDHQ@gmail.com \\ 3 Sardar Begum Dental College, Ghandhara University, Peshawar 25000, Pakistan; kainat.farooq97@gmail.com \\ 4 Natural Products \& Food Research and Analysis (NatuRA), Department of Pharmaceutical Sciences, \\ University of Antwerp, Universiteitsplein 1, 2610 Antwerp, Belgium; kenn.foubert@uantwerpen.be \\ * Correspondence: luc.pieters@uantwerpen.be
}

check for updates

Citation: Rafey, A.; Amin, A.; Kamran, M.; Haroon, U.; Farooq, K.; Foubert, K.; Pieters, L. Analysis of Plant Origin Antibiotics against Oral Bacterial Infections Using In Vitro and In Silico Techniques and Characterization of Active Constituents. Antibiotics 2021, 10 1504. https://doi.org/10.3390/ antibiotics10121504

Academic Editor: Martina Hrast

Received: 21 October 2021 Accepted: 25 November 2021 Published: 8 December 2021

Publisher's Note: MDPI stays neutral with regard to jurisdictional claims in published maps and institutional affiliations.

Copyright: (c) 2021 by the authors. Licensee MDPI, Basel, Switzerland. This article is an open access article distributed under the terms and conditions of the Creative Commons Attribution (CC BY) license (https:// creativecommons.org/licenses/by/ $4.0 /)$.

\begin{abstract}
The pervasiveness of oral bacterial infections in diabetic patients is a serious health concern that may produce severe complications. We investigated 26 Ayurvedic medicinal plants traditionally used for treatment of the oral bacterial infections with the aim to look for new promising drug leads that can be further employed for herbal formulation design. The plants were grouped into three categories based on traditional usage. All plant extracts were examined for antibacterial, antibiofilm and antiquorum-sensing properties. The plants with significant activities including Juglans regia, Syzygium aromaticum, Eruca sativa, Myristica fragrans, Punica granatum and Azadirachta indica were further analyzed using HPLC-DAD-QToF and GC-MS. In silico and in vitro activity was evaluated for selected constituents. Finally, it could be concluded that eugenol and 2-phenylethylisothiocyanate are major contributors towards inhibition of bacterial biofilms and quorum sensing.
\end{abstract}

Keywords: Ayurveda; medicinal plants; biofilm; oral cavity; diabetes mellitus; quorum-sensing; dental plaque

\section{Introduction}

The oral bacterial infections include a wide array of inflammatory conditions that upset various auxiliary structures of the teeth including the periodontal ligament, gingiva and bone. This condition finally leads to loss of teeth with systemic inflammation as seen in periodontitis [1]. Most of isolated bacteria isolated from oral cavity belong to the genera Prophyromonas, Enterococcus, Campylobacter, Bacteroides, Streptococcus, Actinomyces, Staphylococcus, Eubacterium, Lactobacillus, Leptotrichia, Treponema and Fusobacterium [2,3] Some common bacteria isolated from oral cavity include Staphylococcus epidermidis, Staphylococcus aureus (dental plaque) [4,5] Fusobacterium nucleatum, Streptococcus mutanTannerella forsythia, Fusobacterium spp., Prevotella nigrescens, Prevotella intermedia, and Porphyromonas gingivalis [6,7] More importantly, significant changes in the oral environment like orthodontic appliances, $\mathrm{pH}$ changes, antibiotics usage, diabetes etc. upset the microbial homeostasis that may lead to periodontitis and dental caries [8].

Diabetes mellitus is a predominant systemic disease that influences periodontitis to a great extent [9]. Existing statistics provide a strong indication towards interactions of diabetes, gingivitis and periodontitis. The oral cavity possesses an exclusive environment which generally facilitates the growth of an array of microorganisms by providing a diverse 
supply of nutrients, oxygen and humidity [10]. The presence of soft (gingiva) and nonshedding hard (teeth) tissues offers a prospective site for adherence and consequent contact of microorganisms with various host cells [11]. Just after cleaning the teeth, the natural fluid present in the oral cavity causes adsorption of a thin acquired pellicle (composed of saliva glycoproteins including statherin, $\alpha$-amylase, proline-rich proteins, agglutinin and mucins) [12], which induces a change in free energy and surface charge that facilitates bacterial adherence to produce biofilms [13]. The biofilm mass increases by a continuous growth and consequent adsorption of other bacterial species through co-aggregation. It is well documented that pathogens possess a quorum-sensing (QS) controlled ability to form biofilms, which leads to severe infections [14]. QS is termed as the ability of bacteria to perceive and respond rapidly to changes in cell density [15], with the help of small secreted signaling molecules known as auto-inducers. Several QS-based factors, such as bio-surfactant production, exopolysaccharide (EPS) production, swimming and swarming motility have been associated with biofilm formation.

Plant-derived compounds have a long history of treating microbial infections [16] and have received ample attention as a source of anti-QS compounds for the inhibition of biofilms [17]. In traditional medicinal systems including Ayurveda, medicinal plants like Azadirachta indica, Juglans regia, Salvadora persica, Syzygium aromaticum Myristica fragrans and Punica granatum (Table 1) are used that have a long history of medical use in humans. Such medicinal plants or their formulations are being also explored for the purpose of controlling biofilms, due to their non-toxic nature [18].

In current times, in silico tools are commonly used for prediction of drug binding to target site. Generally, drug leads are found using a docking algorithm that helps to classify the optimal binding mode of a drug at the active site of a target molecule. The transcriptional regulators 2Q0J and 3QP1 are used for bacterial biofilm and Quorum sensing in molecular docking analysis to predict possible binding affinities of constituents. This project mainly focuses on the assessment of Ayurvedic medicinal plants for antiquorumsensing and biofilm-inhibiting properties towards clinical strains isolated from diabetic patients with periodontal disease, and characterization of their active constituents. We intended to use the findings of this project for the development of herbal formulation.

Table 1. Traditional uses of selected Ayurvedic medicinal plants.

\begin{tabular}{|c|c|c|}
\hline Plant Species & Ayurvedic/Traditional Usage & References \\
\hline \multicolumn{3}{|c|}{ Category A (Chewing sticks) } \\
\hline Azadirachta indica A. Juss & Antibacterial, antibiofilm & [19] \\
\hline Olea europaea L. & Antibacterial, antibiofilm & [20] \\
\hline Citrullus colocynthis (L.) Schrad. & Antibacterial & [21] \\
\hline Echinops echinatus Roxb. & Antibacterial, & [22] \\
\hline Juglans regia L. ${ }^{1}$ & Antibacterial & {$[23,24]$} \\
\hline Juglans regia L. ${ }^{2}$ & $\begin{array}{l}\text { Antibacterial, Stem and bark } \\
\text { used for teeth cleaning as } \\
\text { chewing sticks in Pakistan } \\
\text { and India }\end{array}$ & {$[23,24]$} \\
\hline Salvadora persica L. ${ }^{3}$ & Antibacterial, antidiabetic & {$[19,25]$} \\
\hline Salvadora persica L. ${ }^{4}$ & Antibacterial, antidiabetic & {$[19,25]$} \\
\hline \multicolumn{3}{|c|}{ Category B (Oils) } \\
\hline Azadirachta indica A. Juss & Antibacterial, antibiofilm & [26] \\
\hline Brassica nigra (L.) K. Koch & For oil pulling & [27] \\
\hline Eruca sativa Mill. & For oil pulling & [28] \\
\hline Lagenaria siceraria (Molina) Standl. & For oil pulling & Local trad. use \\
\hline Phyllanthus emblica L. & For oil pulling & [29] \\
\hline Prunus dulcis Mill. ex Rchb. & For oil pulling & {$[30,31]$} \\
\hline Psoralea corylifolia $\mathrm{L}$. & For oil pulling & {$[32]$} \\
\hline Syzygium aromaticum (L.) Merr. \& L.M. Perry & Antibacterial & [33] \\
\hline
\end{tabular}


Table 1. Cont.

\begin{tabular}{|c|c|c|}
\hline Plant Species & Ayurvedic/Traditional Usage & References \\
\hline \multicolumn{3}{|c|}{ Category C (extracts) } \\
\hline Allium sativum L. $^{5}$ & Antibacterial, antibiofilm & [34] \\
\hline Allium sativum L. 6 & Antibacterial, antibiofilm & [35] \\
\hline Anacyclus pyrethrum (L.) Lag & Antibacterial, antibiofilm & [36] \\
\hline Calotropis procera (Aiton) Dryand & Antibacterial, antibiofilm & [37] \\
\hline Centella asiatica (L.) Urb & Antibacterial, antibiofilm & [38] \\
\hline Illicium verum Hook.f. & Antibacterial, antibiofilm & [39] \\
\hline Myristica fragrans Houtt. ${ }^{7}$ & Antibacterial, antibiofilm & [40] \\
\hline Myristica fragrans Houtt. 8 & Antibacterial, antibiofilm & [40] \\
\hline Punica granatum L. & Antibacterial, antibiofilm & [41] \\
\hline Terminalia arjuna (Roxb. ex DC) Wight \& Arn. & Antibacterial, antibiofilm & [42] \\
\hline Urtica dioica L. & Antibacterial, antibiofilm & [43] \\
\hline Wrightia tinctoria R.Br. & Antibacterial, antibiofilm & [44] \\
\hline
\end{tabular}

${ }^{1}$ Stem peel; ${ }^{2}$ root peel; ${ }^{3}$ origin Saudi Arabia; ${ }^{4}$ origin Pakistan (different origins were screened because of local believes); ${ }^{5}$ black garlic; ${ }^{6}$ garlic; ${ }^{7}$ seed; ${ }^{8}$ mace.

\section{Materials and Methods}

\subsection{Bacterial Strains, Growth Media and Chemicals}

The bacteria isolated from dental plaques were identified as Staphylococcus epidermidis and Staphylococcus aureus (Specimen deposited in Pakistan culture bank), whereas the commercial strains used during investigation included Staphylococcus aureus (ATCC 33862), Chromobacterium violaceum (DSM 30191) and Pseudomonas aeruginosa (ATCC 15442). The bacterial growth media used included, Tryptic Soya Broth (TSB), nutrient agar (Hi Media, India) Luria-Bertani Broth (LB) (Oxoid). The standard compounds were purchased commercially, including juglone (Santa Cruz Biotechnology, Santa Cruz, CA, USA), eugenol (Fluka Honeywell, Seelze, Germany), quercetin (Sigma Aldrich, St. Louis, MO, USA), trans-caryophyllene (Fluka Honeywell, Seelze, Germany), ciprofloxacin (Sigma Aldrich, St. Louis, MO, USA) and azithromycin (Sigma Aldrich, St. Louis, MO, USA), quercitrin (Sigma Aldrich, Steinheim, Germany), 2-phenylisothiocyanate (Sigma Aldrich, Steinheim, Germany), $\alpha$-humulene (Extrasynthese, Genay, France), caryophyllene-oxide (Fluka Honeywell, Seelze, Germany) and apigenin (Sigma Aldrich, Steinheim, Germany).

\subsection{Isolation and Sequencing of Bacteria}

The approval from Ethical review board, Gomal University, D.I. Khan was obtained (2019). Ten patients with periodontitis were enrolled in the investigation and dentist obtained dental plaques (with informed consent) using sterilized techniques. Bacteria were isolated and purified using standard microbiological techniques and biofilm production was initially checked using Congo red agar method [45]. The PCR-based identification was performed in National Culture Collection of Pakistan (NCCP) using 16S rRNA gene sequencing [46]. A modified method [47] (Supplementary Materials) was used for genomic DNA extraction from bacteria, followed by agarose gel electrophoresis and PCR (polymerase chain reaction) [48] (Supplementary Materials) was used. The genes alignment was performed for exact match with NCBI nucleotide database by using nBLAST (Supplementary Materials) and strains were identified as Staphylococcus aureus and Staphylococcus epidermidis. The antibacterial activity of all selected plants was checked against both Staphylococos epidermidis and Staphylococcus aureus (clinical isolates) and Pseudomonas aureginosa and Staphylococos aureus (ATCC strains).

\subsection{Plant Material}

Plant species investigated here are listed in Table 1. Plant material was obtained from authorized herbal medicine stores. The extracts were prepared by cold maceration (90\% methanol) for 10 days (repeated three times) and further liquid-liquid fractionation was accomplished using a rotary evaporator $\left(40{ }^{\circ} \mathrm{C}\right)$. The dried plant material was 
stored at $-4{ }^{\circ} \mathrm{C}$ until further usage. Contrarily, the essential oils were obtained using a Clevenger type apparatus (hydro-distillation) and fixed oils were obtained using the cold press method.

\subsection{Chromatographic Analysis}

\subsubsection{HPLC-DAD Analysis}

A detailed HPLC analysis of extracts was performed using Agilent ${ }^{\circledR} 1200$ series system (HPLC-DAD, Agilent Technologies, Santa Clara, CA, USA). The samples $(10 \mu \mathrm{L})$ were injected at a flow rate of $1 \mathrm{~mL} / \mathrm{min}$ with acetonitrile: water $(0.1 \%$ formic acid) gradient (5\% Acetonitrile to $100 \%$ in $40 \mathrm{~min}$ ). Samples were prepared in a concentration range from 1 to $10 \mathrm{mg} / \mathrm{mL}$ in methanol. A Phenomenex Luna C18 column (silica-based, $250 \times 4.6 \mathrm{~mm}$, $5 \mu \mathrm{m}$ ) (Phenomenex, Torrence, CA, USA) was used.

\subsubsection{HPLC-DAD-QToF Analysis}

The MS 2 analysis of plant extracts was performed by using QToF spectrometer (Xevo G2-XS QToF spectrometer, Waters, Milford, MA, USA), coupled with a LC system (Acquity). The samples were injected $(5 \mu \mathrm{L})$ with a flow rate of $0.6 \mathrm{~mL} / \mathrm{min}$ using a reverse phase column (BEH Shield, $100 \mathrm{~mm} \times 2.10 \mathrm{~mm}, 1.7 \mu \mathrm{m}$, Waters, Milford, MA, USA). A gradient (40 min) mobile phase $\left(\mathrm{H}_{2} \mathrm{O}+0.1 \%\right.$ formic acid and Acetonitrile $\left.+0.1 \% \mathrm{FA}\right)$ was used. The DAD spectra were recorded between 190 and $500 \mathrm{~nm}$. A full scan data (ESI [-] and ESI $[+], m / z 50$ to 1500) was recorded using sensitivity mode (approximate resolution: 22,000 FWHM) during first scan. Other parameters were source, desolvation temperature at $120^{\circ} \mathrm{C}$ and $550{ }^{\circ} \mathrm{C}$, spray voltage was $+1.0 \mathrm{kV}$ and $-0.8 \mathrm{kV}$; cone gas flow $50.0 \mathrm{~L} / \mathrm{h}$, and desolvation gas flow $1000.0 \mathrm{~L} / \mathrm{h}$, respectively.

\subsubsection{GC-MS Analysis}

The essential oil analysis was accomplished by using GC-MS (Thermo Scientific, WM, USA) with an (AOC-20i) and FID detector (flame ionization detector). The sample was injected $(0.5 \mu \mathrm{L})$ and Helium $(1 \mathrm{~mL} / \mathrm{min})$ was used as carrier gas. The DB-5 MS capillary column with $(30 \mathrm{~m} \times 0.25 \mathrm{~mm})$ with $0.25 \mu \mathrm{m}$ film thickness was used. The column oven temperature was initially set at $40-90{ }^{\circ} \mathrm{C}\left(2{ }^{\circ} \mathrm{C} / \mathrm{min}\right)$ that was upraised to $90-240{ }^{\circ} \mathrm{C}$ $\left(3{ }^{\circ} \mathrm{C} / \mathrm{min}\right)$. The temperature of injector and detector was kept constant at $240{ }^{\circ} \mathrm{C}$ and $280^{\circ} \mathrm{C}$, correspondingly. The Trace GC (Ultra DSQ) was used for identification in electron ionization mode operating at $70 \mathrm{eV}$. The mass units were monitored from 35 to $500 \mathrm{amu}$. A capillary column (AT-5MS, Grace) dimensions $30 \mathrm{~m} \times 0.25 \mathrm{~mm}$ with $0.25 \mu \mathrm{m}$ film thickness was used using same temperature conditions as GC analysis. The components identification was performed using comparison with NIST library.

\subsection{Molecular Docking}

The molecular docking studies were performed using AutoDock vina v 4.2. In the first part, structures (X-ray crystallographic) of the transcriptional regulators 2Q0J [49] and 3QP1 [50] were downloaded from the Protein Data Bank (PDB). In subsequent part, the 3D structures of test samples (SDF format) were obtained from Pubchem database. Then the PDB format files were created by using Accelrys DS Visualizer 2.0 [51]. The active pocket dimensions for targets were recorded by using CASTp 3.0, followed by compulsory processing for removal of water molecules and $\mathrm{H}$ atom, charges addition. Finally, molecular docking was performed using Lamarckian Genetic Algorithm embedded in AutoDock v 4.2. [52]. A total number of 45 poses were generated and grouped according to their RMSD and best docked molecules $[\Delta \mathrm{G}]$ were analyzed using Ligplot ${ }^{+}$Accelrys DS Visualizer 2.0 and PYMOL. 


\subsection{Biological Activities}

2.6.1. Determination of MIC and MBC (Minimum Inhibitory and Bactericidal Concentrations)

The antimicrobial assays were performed with the help of a modified protocol [53]. To the 96 microwell plates (NEST, China), $24 \mathrm{~h}$ old bacterial cultures $\left(50 \mu \mathrm{L}, 1.5 \times 10^{7} \mathrm{CFU} / \mathrm{mL}\right.$ ) and test sample ( $50 \mu \mathrm{L}$, various dilutions) were added. The plates were then incubated at $37^{\circ} \mathrm{C}$ for $24 \mathrm{~h}$ and afterwards resazurin $(40 \mu \mathrm{L}, 0.015 \%)$ solution was added and incubated further for $60 \mathrm{~min}\left(37^{\circ} \mathrm{C}\right)$. A 96-microplate reader (Hippo MPP-96, Biosan) was used for recording results. For $\mathrm{MBC}$ determination, $10 \mu \mathrm{L}$ of bacterial suspensions was collected from MIC microwells and streaked on Muller Hinton agar plates and incubated for $24 \mathrm{~h}$ at $37^{\circ} \mathrm{C}$. The growth on Muller Hinton agar plates was monitored by counting colonies. Ciprofloxacin was used as reference standard.

\subsubsection{Antibiofilm Activity}

A modified method for inhibition of biofilm formation was used during this study [54]. Briefly, 24 hrs old bacterial cultures in TSB media (adjusted with $0.5 \mathrm{McFarland}$ ) and $100 \mu \mathrm{L}$ of test sample $(0.01-3 \mathrm{mg} / \mathrm{mL}$ ) were added to 12 -well polystyrene plates and incubated for $24 \mathrm{~h}$ at $37^{\circ} \mathrm{C}$. Afterwards, the cell growth in the plates was monitored at $592 \mathrm{~nm}$. For quantification, the crystal violet staining of biofilms (in 12-well plates) was performed, followed by addition of $95 \%$ ethanol to stained cells. Finally, the absorbance (Abs) was recorded at $592 \mathrm{~nm}$ for quantification of biofilm formation. The \% inhibition was calculated using the following formula:

$$
\% \text { Inhibition }=[1-(\text { OD of test sample } / \text { OD of control })] \times 100
$$

\subsubsection{Antiquorum-Sensing}

The anti QS potential of compounds was determined by using the previously described procedure [55]. The BHIA (Brain Heart Infusion Agar) was inoculated with an overnight bacterial culture (C. violaceum, 1/100 ratio) followed by placing sterilized paper discs $(6 \mathrm{~mm})$. The test sample $(12 \mu \mathrm{L}, 0.01-3 \mathrm{mg} / \mathrm{mL})$ was added on each disc and kept to dry for $40 \mathrm{~min}$. Later, the seeded plates were incubated at $30^{\circ} \mathrm{C}$ for 3 days. The results were determined by recording the zone of inhibition around discs. Ciprofloxacin was used as reference drug.

\subsubsection{Violacein Inhibition Assay}

For quantification of violacein produced by C. violaceum, an improved procedure was used [56]. Briefly, an overnight grown culture $(200 \mu \mathrm{L})$ of $C$. violaceum (OD $=0.4$ at $600 \mathrm{~nm}$ ) was added to sterilized microtiter plates containing various concentrations of test samples $(1-4 \mathrm{mg} / \mathrm{mL})$. The plates were kept at $30^{\circ} \mathrm{C}$ for $24 \mathrm{~h}$ and monitored for inhibition of violacein production by observing the absorbance at $585 \mathrm{~nm}$. The \% of inhibition was calculated by using the following formula:

$$
\% \text { Violacein inhibition }=[1-(\mathrm{OD} \text { test sample } / \mathrm{OD} \text { control })] \times 100
$$

\section{Results and Discussion}

\subsection{Screening of Plant Extracts}

Initially a detailed survey of Ayurvedic medicinal plants reported to possess either antibacterial or antibiofilm activity, or used for oral cavity problems, was performed. A diverse collection of traditional medicinal plants was employed. The plants were categorized as category A (miswak or chewing sticks, commonly employed for oral hygiene), category B (oils, that have been used for oil pulling in traditional Ayurveda) and category C (traditional antimicrobial plants that have Ayurvedic claims for rinsing of the oral cavity) (Table 1). Initially the biofilm producing strains were tested against commonly prescribed 
antibiotics and antibiograms were recorded (Table 2). It was clear from these results that most of the tested antibiotics were resistant to clinical strains.

Table 2. Antibiogram profiles of tested strains.

\begin{tabular}{|c|c|c|c|c|c|c|c|c|c|c|c|c|c|c|c|}
\hline $\begin{array}{c}\text { Bacterial } \\
\text { Strain }\end{array}$ & 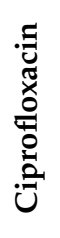 & 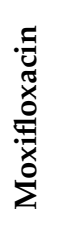 & 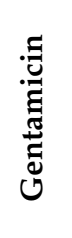 & 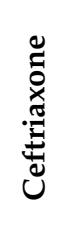 & 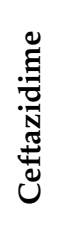 & :ี & 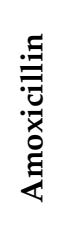 & 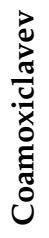 & 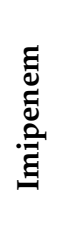 & 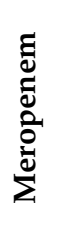 & 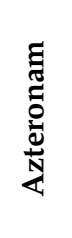 & 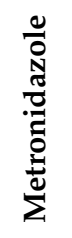 & 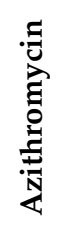 & 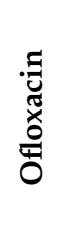 & 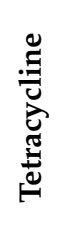 \\
\hline $\begin{array}{l}\text { Staphylococcus } \\
\text { aureus }{ }^{1}\end{array}$ & 20 & 80 & 100 & 100 & 100 & 100 & 90 & 80 & 60 & 60 & 100 & 100 & 100 & 90 & 90 \\
\hline $\begin{array}{l}\text { Staphylococcus } \\
\text { epidermidis }{ }^{1}\end{array}$ & 20 & 90 & 100 & 100 & 100 & 100 & 100 & 80 & 60 & 60 & 100 & 100 & 100 & 100 & 100 \\
\hline $\begin{array}{l}\text { Staphylococcus } \\
\text { aureus }^{2}\end{array}$ & 10 & 0 & 60 & 40 & 40 & 20 & 30 & 10 & 0 & 0 & 100 & 100 & 0 & 40 & 40 \\
\hline $\begin{array}{l}\text { Pseudomonas } \\
\text { aureginosa }^{2}\end{array}$ & 10 & 0 & 100 & 40 & 40 & 20 & 40 & 10 & 0 & 0 & 100 & 100 & 0 & 50 & 30 \\
\hline
\end{tabular}

In plant species from category $\mathrm{A}$, in general the better inhibition against $S$. aureus (ATCC) with significant MIC values (ranging from 0.012 to $0.097 \mathrm{mg} / \mathrm{mL}$ ) (Table 3) was observed. Overall, the Juglans regia extracts (root peel and stem peel) were able to produce significant inhibition $(0.048-0.78 \mathrm{mg} / \mathrm{mL})$ against the clinical isolate of S. aureus. Likewise, the category A plants also showed better inhibition against Pseudomonas aureginosa (ATCC) at diverse MIC values (ranging from $0.024-1.2 \mathrm{mg} / \mathrm{mL}$ ). In the case of $S$. epidermidis (clinical isolate), most plant extracts showed no inhibition; however, the Juglans regia extract presented significant inhibition $(0.048-0.78 \mathrm{mg} / \mathrm{mL})$. During initial anti-biofilm analysis, Juglans regia extracts showed a moderate inhibition (52\% at $200 \mu \mathrm{g} / \mathrm{mL}$ ) (Table 4$)$. All other plant species from category A either showed no inhibition or presented low levels of inhibition $(<50 \%)$. Based on the antibacterial properties, the plant species with significant activity were further processed in anti-biofilm and anti-QS assays. A moderate anti-QS activity (5-7 mm zone of inhibition and 51\% inhibition of violacin) was noticed for Juglans regia extracts. These results are in compliance with the obtained MICs. Literature has suggested that active ingredients present in Juglans regia may include juglone, regiolone, glansreginin A, glansreginin B and proceroside [57-59] (Table 5), that may be contributing to inhibition.

Table 3. Determination of MIC values $(\mathrm{mg} / \mathrm{mL})$ of tested plant species.

\begin{tabular}{|c|c|c|c|c|}
\hline Plant Species & S. aureus ${ }^{\text {a }}$ & S. epidermidis ${ }^{\mathbf{b}}$ & S. aureus ${ }^{\mathrm{c}}$ & p. aerugionasa ${ }^{\mathrm{d}}$ \\
\hline Allium satioum ${ }^{1}$ & $>100$ & $>100$ & $>100$ & 1.2 \\
\hline Allium sativum $^{2}$ & $>100$ & $>100$ & 0.6 & 0.6 \\
\hline Anacyclus pyrethrum & $>100$ & 1.2 & $>100$ & 1.2 \\
\hline Azadirachta indica & $>100$ & 0.78 & 0.024 & 0.024 \\
\hline Azadirachta indica oil & 0.39 & $>100$ & 0.09 & 0.78 \\
\hline Brassica nigra oil & $>100$ & $>100$ & $>100$ & $>100$ \\
\hline Calotropis procera & $>100$ & $>100$ & 1.2 & 1.2 \\
\hline Centella asiatica & $>100$ & $>100$ & 1.2 & 0.048 \\
\hline Citrullus colocynthis & $>100$ & $>100$ & 0.012 & $>100$ \\
\hline Echinops echinatu & $>100$ & $>100$ & 0.024 & $>100$ \\
\hline Eruca sativa oil & 0.012 & 0.097 & 0.024 & 0.012 \\
\hline Illicium verum & 1.2 & 0.6 & 0.048 & 0.097 \\
\hline Juglans regia ${ }^{3}$ & 0.78 & 0.048 & 0.097 & 0.048 \\
\hline Juglans regia 4 & 0.048 & 0.78 & 0.097 & 0.097 \\
\hline Lagenaria siceraria oil & $>100$ & $>100$ & $>100$ & $>100$ \\
\hline
\end{tabular}


Table 3. Cont.

\begin{tabular}{|c|c|c|c|c|}
\hline Plant Species & S. aureus ${ }^{\text {a }}$ & S. epidermidis ${ }^{\mathbf{b}}$ & S. aureus ${ }^{\mathrm{c}}$ & p. aerugionasa ${ }^{\mathrm{d}}$ \\
\hline Myristica fragrans 5 & 0.097 & 0.024 & 0.097 & 0.024 \\
\hline Myristica fragrans 6 & 0.097 & 0.048 & 0.78 & 0.048 \\
\hline Olea europaea & $>100$ & 1.2 & 0.048 & 0.048 \\
\hline Phyllanthus emblica oil & $>100$ & $>100$ & $>100$ & $>100$ \\
\hline Prunus dulcis oil & $>100$ & $>100$ & $>100$ & $>100$ \\
\hline Psoralea corylifolia oil & $>100$ & $>100$ & $>100$ & $>100$ \\
\hline Punica granatum & 0.78 & 0.39 & 0.19 & 0.39 \\
\hline Salvadora persica ${ }^{7}$ & $>100$ & $>100$ & 0.024 & 1.2 \\
\hline Salvadora persica ${ }^{8}$ & $>100$ & 1.2 & 0.012 & 1.2 \\
\hline Sesamum radiatum & $>100$ & $>100$ & $>100$ & $>100$ \\
\hline Syzygium aromaticum oil & 0.024 & 0.097 & 0.024 & 0.024 \\
\hline Terminalia arjuna & $>100$ & $>100$ & 0.78 & 0.78 \\
\hline Urtica dioica & $>100$ & $>100$ & $>100$ & 1.2 \\
\hline Wrightia tinctoria & $>100$ & $>100$ & 0.78 & 1.56 \\
\hline Ciprofloxacin & $<0.004$ & $<0.004$ & 0.009 & 0.009 \\
\hline Azithromycin & $>100$ & $>100$ & 0.008 & 0.002 \\
\hline
\end{tabular}

Table 4. Antibiofilm and antiquorum-sensing activity of plant extracts and essential/fixed oils.

\begin{tabular}{|c|c|c|c|}
\hline Plant Species & $\begin{array}{c}\text { Inhibition of } \\
\text { Bacterial Biofilm * }\end{array}$ & Zone of Inhibition ** & Violacein Inhibition \\
\hline Allium sativum $^{1}$ & $\mathrm{Nil}^{\mathrm{a}}$ & $0^{\mathrm{a}}$ & $\mathrm{Nil}^{\mathrm{a}}$ \\
\hline Allium satioum $^{2}$ & $\mathrm{Nil}^{\mathrm{a}}$ & $0^{\mathrm{a}}$ & $\mathrm{Nil}^{\mathrm{a}}$ \\
\hline Anacyclus pyrethrum & $\mathrm{Nil}^{\mathrm{a}}$ & $0^{\mathrm{a}}$ & $\mathrm{Nil}^{\mathrm{a}}$ \\
\hline Azadirachta indica & $\mathrm{Nil}^{\mathrm{a}}$ & $0^{\mathrm{a}}$ & $\mathrm{Nil}^{\mathrm{a}}$ \\
\hline Azadirachta indica oil & $54 \%$ a & $6^{\mathrm{a}}$ & $54 \%$ a \\
\hline Brassica nigra oil & $\mathrm{Nil}^{\mathrm{a}}$ & $0^{\mathrm{a}}$ & $\mathrm{Nil}^{\mathrm{a}}$ \\
\hline Calotropis procera & $25 \%$ a & $0^{\mathrm{a}}$ & $\mathrm{Nil}^{\mathrm{a}}$ \\
\hline Centella asiatica & $28 \%{ }^{a}$ & $2^{a}$ & $26 \%{ }^{a}$ \\
\hline Citrullus colocynthis & $\mathrm{Nil}^{\mathrm{a}}$ & $0^{\mathrm{a}}$ & $\mathrm{Nil}^{\mathrm{a}}$ \\
\hline Echinops echinatus & $\mathrm{Nil}^{\mathrm{a}}$ & $0^{\mathrm{a}}$ & $\mathrm{Nil}^{\mathrm{a}}$ \\
\hline Eruca sativa oil & $58 \% \mathrm{~d}$ & $14^{\mathrm{d}}$ & $52 \% d$ \\
\hline Illicium verum & $38 \%^{a}$ & $2^{a}$ & $35 \%{ }^{a}$ \\
\hline Juglans regia ${ }^{3}$ & $51 \%$ b & $5^{b}$ & $50 \%$ b \\
\hline Juglans regia 4 & $52 \%$ b & $7^{b}$ & $51 \% \mathrm{~b}$ \\
\hline Lagenaria siceraria oil & $\mathrm{Nil}^{\mathrm{a}}$ & $0^{\mathrm{a}}$ & $\mathrm{Nil}^{\mathrm{a}}$ \\
\hline Myristica fragrans 5 & $39 \%$ a & $2^{a}$ & $35 \%$ a \\
\hline Myristica fragrans 6 & $35 \%$ a & $2^{a}$ & $32 \%$ a \\
\hline Olea europaea & $28 \%$ a & $2^{a}$ & $31 \%$ a \\
\hline Phyllanthus emblica oil & $\mathrm{Nil}^{\mathrm{a}}$ & $0^{\mathrm{a}}$ & $\mathrm{Nil}^{\mathrm{a}}$ \\
\hline Prunus dulcis oil & $\mathrm{Nil}^{\mathrm{a}}$ & $0^{\mathrm{a}}$ & $\mathrm{Nil}^{\mathrm{a}}$ \\
\hline Psoralea corylifolia oil & $\mathrm{Nil}^{\mathrm{a}}$ & $0^{\mathrm{a}}$ & $\mathrm{Nil}^{\mathrm{a}}$ \\
\hline Punica granatum & $55 \%$ a & $5^{a}$ & $52 \%$ a \\
\hline Salvadora persica ${ }^{7}$ & $\mathrm{Nil}^{\mathrm{a}}$ & $0^{\mathrm{a}}$ & $\mathrm{Nil}^{\mathrm{a}}$ \\
\hline Salvadora persica 8 & $\mathrm{Nil}^{\mathrm{a}}$ & $0^{\mathrm{a}}$ & $\mathrm{Nil}^{\mathrm{a}}$ \\
\hline Sesamum radiatum & $\mathrm{Nil}^{\mathrm{a}}$ & $0^{\mathrm{a}}$ & $\mathrm{Nil}^{\mathrm{a}}$ \\
\hline Syzygium aromaticum oil & $72 \%^{c}$ & $16^{c}$ & $61 \%{ }^{c}$ \\
\hline Terminalia arjuna & $41 \%$ a & $2^{a}$ & $38 \%$ a \\
\hline Urtica dioica & $42 \%$ a & $2^{\mathrm{a}}$ & $41 \%$ a \\
\hline Wrightia tinctoria & $\mathrm{Nil}^{\mathrm{a}}$ & $0^{\mathrm{a}}$ & $\mathrm{Nil}^{\mathrm{a}}$ \\
\hline
\end{tabular}

${ }^{1}$ Black garlic; ${ }^{2}$ garlic; ${ }^{3}$ stem peel; ${ }^{4}$ root peel; ${ }^{5}$ seed; ${ }^{6}$ mace; ${ }^{7}$ origin Saudi Arabia; ${ }^{8}$ Origin Pakistan; ${ }^{\mathrm{a}}=200 \mu \mathrm{g} / \mathrm{mL}$ (max. tested concentration); ${ }^{\mathrm{b}}=70 \mu \mathrm{g} / \mathrm{mL} ;{ }^{\mathrm{c}}=0.19 \mu \mathrm{g} / \mathrm{mL} ;{ }^{\mathrm{d}}=0.39 \mu \mathrm{g} / \mathrm{mL} ;{ }^{*}$ Pseudomonas. aeruginosa $(\%) ;{ }^{* *}$ Chromobacterium violaceum $(\mathrm{mm})$. 
Table 5. Chemical composition of active plants according to literature.

\begin{tabular}{|c|c|}
\hline Plant Species & Major Constituents \\
\hline Azadirachta indica & Nimbidine, azadirachtin (Azadirachtin A), salannol, salannin [60] \\
\hline Eruca sativa & $\begin{array}{l}\text { Bis (4-isothiocyanatobutyl) disulphide }(5000 \mu \mathrm{g} / \mathrm{g}) \text {, sulforaphane } \\
(743 \mu \mathrm{g} / \mathrm{g}), 2 \text {-phenylethyl isothiocyanate }(158 \mu \mathrm{g} / \mathrm{g}) \\
\text { 3-butenyl isothiocyanate }(259.6 \mu \mathrm{g} / \mathrm{g}) \text {, erucic acid }(57 \%)[61]\end{array}$ \\
\hline Illicium verum & $\begin{array}{l}\text { Trans-anethol }(71.98 \%) \text {, feniculine }(14.5 \%) \text { [62] estragole }(1.84 \%) \text {, } \\
\text { limonene }(1.38 \%), 4 \text {-allyl anisole }(6.7 \%) \text { [63] }\end{array}$ \\
\hline Juglans regia & $\begin{array}{l}\text { Juglone, regiolone }(28.6 \%) \text {, proceroside }(9.1 \%) \text { [64] glansreginin B }(11.5 \%) \text {, } \\
\text { glansreginin A }(10.4 \%) \text { [65] quercetin, quercitrin, gallic acid [58] }\end{array}$ \\
\hline Myristica fragrans ${ }^{1}$ & $\begin{array}{l}\text { Myrislignan (22.59\%), elemicin }(13.99 \%), \\
\alpha \text {-phellandrene }(13.04 \%)[66] \\
\text { Sabinene }(28 \%), \beta \text {-pinene }(9.72 \%), \alpha \text {-pinene }(10.2 \%)[67] \text { safrole, eugenol, } \\
\text { caryophyllene, caryophyllene oxide, palmitic acid [68] }\end{array}$ \\
\hline Myristica fragrans ${ }^{2}$ & $\begin{array}{l}\text { Safrole, sabinene (28\%), } \beta \text {-pinene }(9.72 \%) \text {, } \\
\text { eugenol, caryophyllene, caryophyllene oxide, palmitic acid [68] }\end{array}$ \\
\hline Syzygium aromaticum & $\begin{array}{l}\text { Eugenol }(87-89 \%) \text {, caryophyllene }(3.56 \%) \text {, } \\
\text { eugenyl acetate }(8.01 \%), \alpha \text {-humulene }(0.04 \%) \text {, caryophyllene } \\
\text { oxide }(0.47 \%)[11,69]\end{array}$ \\
\hline Punica granatum & $\begin{array}{l}\text { Gallic acid, caffeic acid, chlorogenic acid, ellagic acid, apigenin, quercetin, } \\
\text { pelargonidin, cyanidin, punicalin, punicalagin, granatin A, } \\
\text { granatin B }[59,70]\end{array}$ \\
\hline
\end{tabular}

In plant species from category B (fixed and essential oils), only Syzygium aromaticum $(0.024-0.097 \mathrm{mg} / \mathrm{mL})$ showed promising inhibition against both clinical and reference strains (ATCC) (Table 2). The antibiofilm results reveal the highest inhibition by Syzygium aromaticum ( $72 \%$ at $0.19 \%$ ) (Table 4$)$. The Syzygium aromaticum oil showed an excellent inhibition $(16 \mathrm{~mm})$ of $C$. violaceum with a high $(61 \%)$ violacein inhibition at the tested concentration (Table 4). A detailed literature review has suggested that Syzygium aromaticum oil mainly contains eugenol, caryophyllene, caryophyllene-oxide, eugenyl acetate and $\alpha$-humulene $[11,69]$. Thus, the strong inhibition could possibly be due to a single ingredient or due to an additive or synergistic effect. Eugenol and $\alpha$-humulene have been reported to show strong antimicrobial properties [69]. The Eruca sativa oil $(0.024-0.097 \mathrm{mg} / \mathrm{mL})$ also showed promising inhibition against both clinical and reference strains (ATCC) (Table 3). Additionally, moderate antibiofilm activities (58\%) and anti-QS (14 mm zone of inhibition, $52 \%$ inhibition of violacein) were recorded in the case of Eruca sativa oil (Table 3). The main components of Eruca sativa oil are bis-[4-isothiocyanatobutyl) disulphide, sulforaphane, 2-phenylethyl isothiocyanate, 3-butenyl isothiocyanate and erucic acid [14,61] (Table 5), that may be contributing towards strong inhibition.

In plant species from category C (traditional plants with antimicrobial claims), the majority of the tested extracts were not able to show any reportable activity (maximum tested concentration $1.2 \mathrm{mg} / \mathrm{mL}$ ); however the Myristica fragrans extracts (both seeds and mace) showed remarkable activity (MIC ranging from 0.024 to $0.097 \mathrm{mg} / \mathrm{mL}$ ) (Table 3). With regard to antibiofilm activity, a mild activity was noticed (35-39\%). Additionally, similar trends were seen in anti-QS activity ( $2 \mathrm{~mm}$ zone of inhibition; 32-35\% inhibition of violacein). Researchers have reported that Myristica fragrans is a rich source of sabinene, elemicin, caryophyllene, caryophyllene-oxide, $\alpha$-phellandrene, $\alpha$-pinene and $\beta$-pinene [66,67] (Table 3). Sabinene and elemicin have strong antibacterial properties [68] that can contribute to the observed activity. Likewise, Illicium verum extracts also showed significant activity $(0.048-1.2 \mathrm{mg} / \mathrm{mL})$. However, a mild level of antibiofilm and anti QS activity was recorded (Table 3). The essential oil Illicium verum mainly contains trans-anethol, feniculin [62], estragole, limonene and 4-allylanisole [64] (Table 4). Based on relative abundance, it was concluded that trans-anethol may contribute to the antimicrobial activity [64]. 
The peel extracts of Punica granatum also showed strong inhibition against all tested strains (MIC ranging from $0.19-0.78 \mathrm{mg} / \mathrm{mL}$ ) (Table 3). During antibiofilm activity, a moderate inhibition was noticed (55\%). Similar trends were seen in anti-QS activity (5 mm zone of inhibition; 52\% inhibition of violacein). The peel is commonly known for strong antioxidant properties and is a rich source of punicalagin, punicalin, apigenin, quercetin, kaempferol, gallic acid and ellagic acid [59,70] (Table 5). The presence of phenolics and flavonoids may contribute to strong antibacterial properties.

Lastly, Azadirachta indica seed oil also showed significant antimicrobial (0.09-0.78\%) antibiofilm (54\%) and antiquorum-sensing activities $(6 \mathrm{~mm})$. The A. indica seed oil has been reported to contain nimbidine, azadirachtin (Azadirachtin A), salannol, salannin [60], which possess antimicrobial properties [71]. In our case, the major components were not tested due to the unavailability of compounds.

\subsection{GC-MS and HPLC-DAD-MS-QToF Analysis}

Based on preliminary analysis, the plant samples with significant antibacterial activity were analyzed using HPLC-DAD. Afterwards GC-MS (oils) and HPLC-DAD-MS-QToF analysis (crude extracts) was performed covering all active plants. Chromatographic profiles are available as Supplementary Materials (Figures S1-S13). The results were compared with literature (Table 5), and pure available compounds were screened further to predict their role in the observed activities of plant extracts or oils.

\subsection{Molecular Docking of Compounds}

In the transcriptional regulator 2Q0J, quercitrin showed a nice fit in the binding pocket with pose 9 and free binding energy $-6.6 \Delta \mathrm{G}\left(\mathrm{kJ} \mathrm{mol}^{-1}\right)$ (Table S10). Quercitrin showed strong H-bonding interaction with seven amino acid residues including Asp259, Glu256, Gly255, Gln252, Ser294, Arg295, Ser257 (Figure 1). Likewise, juglone presented a good fitting in the binding pocket with pose 1 and free binding energy $-8.5 \Delta \mathrm{G}\left(\mathrm{kJ} \mathrm{mol}^{-1}\right)$ (Table S10). Juglone displayed strong H-bonding interaction with five amino acid residues including Asp73, Asp 178, His221, His 282, Ser 285 (Figure 1). Likewise, eugenol displayed a good placement in the binding pocket with pose 2 with free binding energy $-6.2 \Delta \mathrm{G}\left(\mathrm{kJ} \mathrm{mol}^{-1}\right)$ (Table S10). A strong H-bonding interaction with three amino acid residues including Asp73, His71, Asp178 was observed (Figure 1). Interestingly, transcaryophyllene and $\alpha$-humulene did not present any $\mathrm{H}$-bonding interactions within the prescribed pocket (Table S10). It was therefore concluded that quercitrin, juglone and eugenol may be contributing towards biological activity.

In the transcriptional regulator 3QP1, gallic acid showed the best fitting (free binding energy $-5.1 \Delta \mathrm{G} \mathrm{kJ} \mathrm{mol}^{-1}$ ) in the binding pocket of this protein with pose rank 1 (Figure 2). The H-bonding interacting residues included Glu113, Glu112, Gly138, Trp111, Gly158 and Arg163 Similarly, quercitrin showed a nice fit in the binding pocket with pose 4 with free binding energy -7.2 $\Delta \mathrm{G}\left(\mathrm{kJ} \mathrm{mol}^{-1}\right)$ (Table S10). Quercitrin showed strong H-bonding interaction with six amino acid residues including Arg59, Gly136, Ser137, Pro52, Glu160 and Arg163 (Figure 2). Likewise, apigenin showed an acceptable arrangement in the binding pocket with pose 3 with free binding energy $-6.4 \Delta \mathrm{G}\left(\mathrm{kJ} \mathrm{mol}^{-1}\right)$ (Table S10). In this case, strong $\mathrm{H}$-bonding interactions were noticed with four amino acid residues including Glu112, Trp111, Gly128, Arg163 (Figure 2). In the case of eugenol pose 6 (free binding energy $-5.0 \Delta \mathrm{G}\left(\mathrm{kJ} \mathrm{mol}^{-1}\right)$ (Table S10) showed a nice fit in the binding pocket of transcriptional regulator 3QP1. A strong $\mathrm{H}$-bonding interaction with three amino acid residues including Trp111, Gly128 and Glu112 was recorded (Figure 2). Trans-caryophyllene and $\alpha$-humulin were unable to present any interaction within the prescribed pocket (Table S10). Thus, it was hypothesized that gallic acid, quercitrin, apigenin and eugenol may contribute towards significant inhibition. Based on docking results of these compounds against all targets it was concluded that the compounds with more H-bonding interactions may contribute towards significant biological activities. Therefore, based on docking results, in vitro analysis of these compounds was performed. 
a

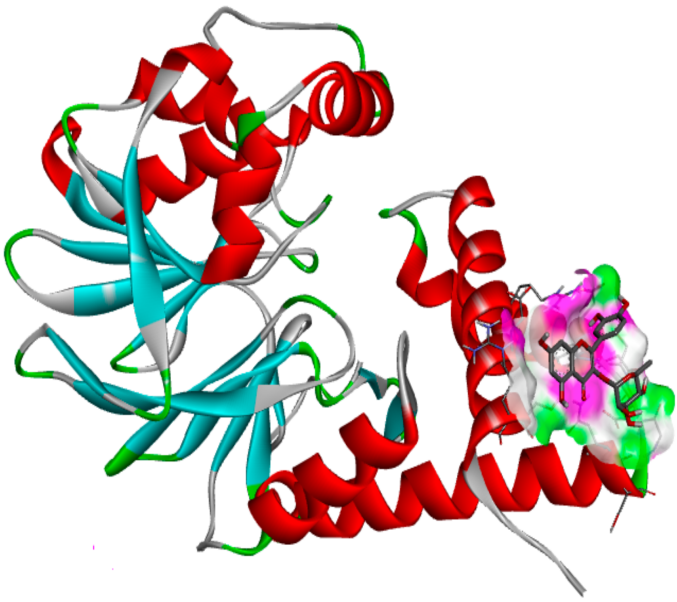

$\mathrm{b}$

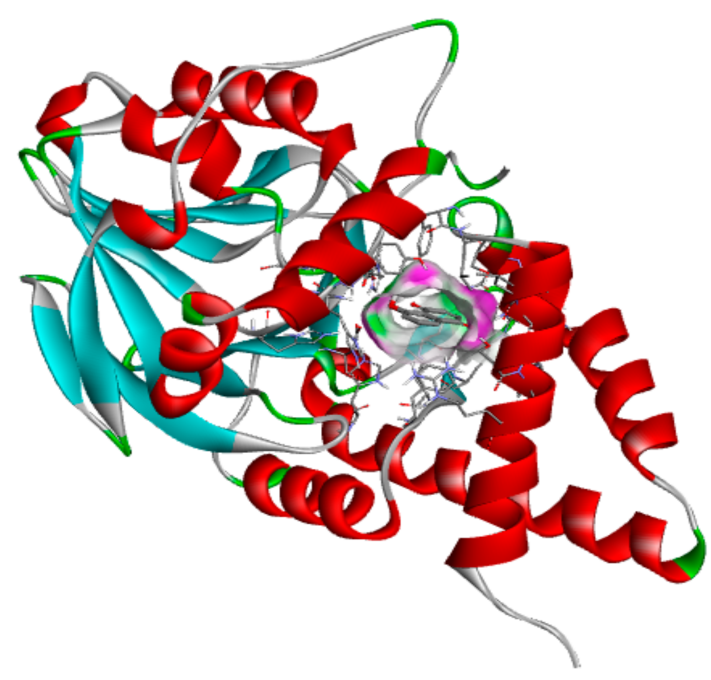

C

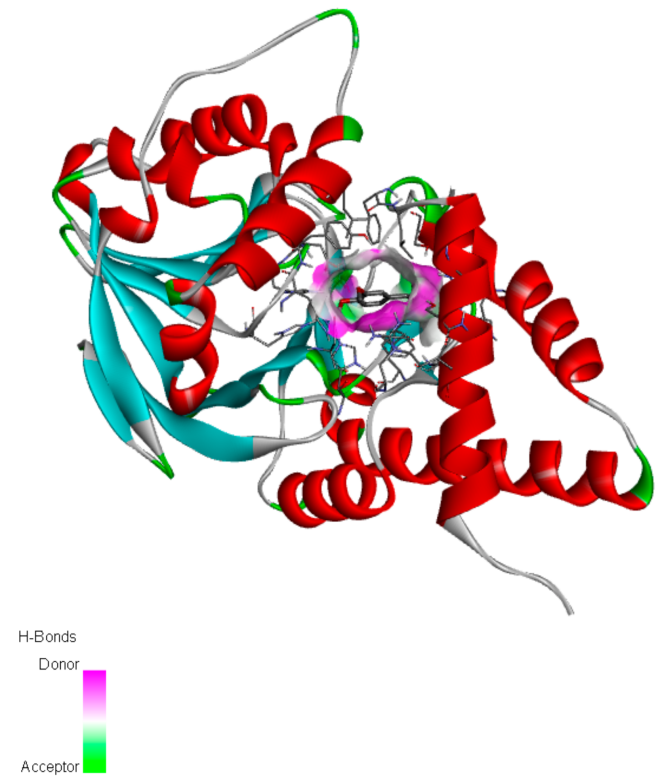

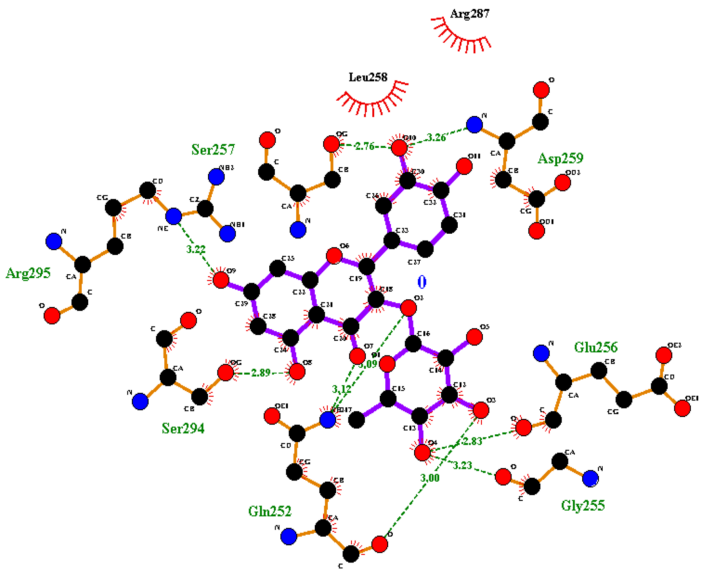
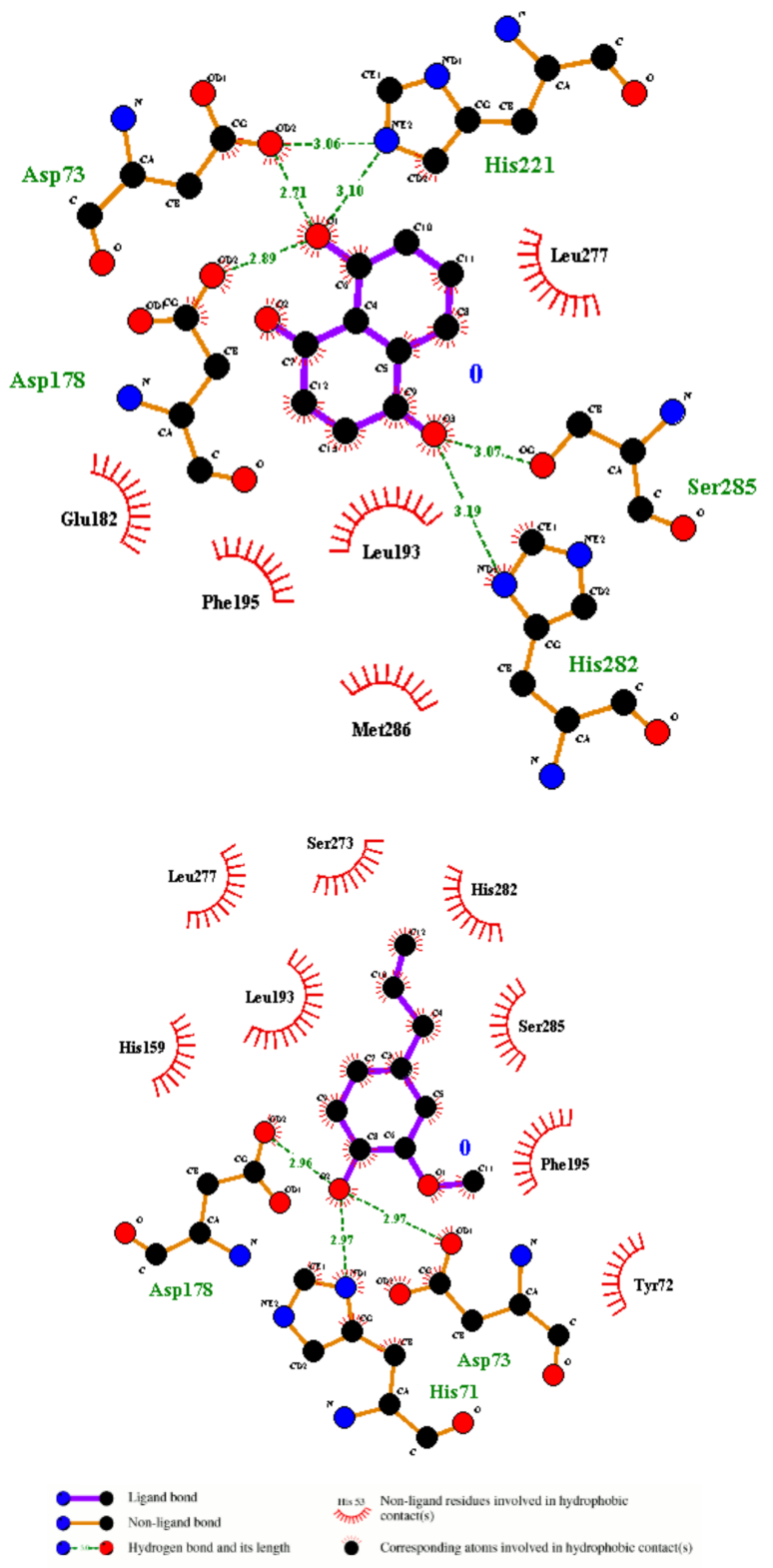

Figure 1. 3D interaction and $\mathrm{H}$, non-H bonding interactions of (a) querccitrin (pose 1) (b) juglone (pose 1) and (c) eugenol (pose 2) inside binding sites of transcriptional regulator 2Q0J. 


\subsection{Biological Activities of Pure Compounds}

The major components of Syzygium aromaticum oil include eugenol, caryophyllene, caryophyllene-oxide and $\alpha$-humulene (Table 5). During antibacterial analysis, a reportable inhibition was recorded against all strains (MIC ranging from 0.0003 to $0.0 .39 \mathrm{mg} / \mathrm{mL}$ ) (Table 6). A significant inhibition of bacterial biofilms (52-55\%) and quorum-sensing (15 mm zone of inhibition and 57.2\% violacein inhibition) was observed (Table 7). Similarly, caryophyllene-oxide showed antibacterial activity (MIC range $0.05-0.75 \mathrm{mg} / \mathrm{mL}$ ) against all tested bacterial strains (Table 6). During antibiofilm assays, poor inhibition (17\%) was recorded against S. epidermidis (clinical strain), whereas a moderate inhibition (51\%) was observed against $S$. aureus (clinical strain). However, a moderate inhibition of quorumsensing ( $4 \mathrm{~mm}$ zone of inhibition; $54 \%$ violacein inhibition) was recorded (Table 7 ). In case of $\alpha$-humulene a moderate antibacterial activity was seen $(0.03-0.18 \mathrm{mg} / \mathrm{mL})$ against all tested strains (Table 6). Similarly, a moderate (56-58\%) antibiofilm activity was recorded against tested strains and only a slight anti-QS activity ( $1 \mathrm{~mm}$ zone of inhibition; $40 \%$ violacein inhibition) was noticed (Table 7).

Table 6. MIC (mg/mL) of pure compounds against clinical and reference strains.

\begin{tabular}{|c|c|c|c|c|}
\hline Compound Name & S. aureus ${ }^{\text {a }}$ & S. epidermidis ${ }^{\mathbf{b}}$ & S. aureus ${ }^{\mathrm{c}}$ & p. aeruginosa ${ }^{\mathrm{a}}$ \\
\hline Juglone & 0.0058 & 0.046 & 0.75 & 0.05 \\
\hline Caryophyllene-oxide & 0.37 & 0.37 & 0.75 & 0.05 \\
\hline$\alpha$-Humulene & 0.023 & 0.37 & 0.75 & 0.187 \\
\hline Eugenol & 0.0003 & 0.0003 & 0.039 & 0.019 \\
\hline 2-Phenylethyl isothiocyanate & 0.0058 & 0.023 & 0.093 & 0.18 \\
\hline Caryophyllene & $>0.75$ & $>0.75$ & 0.75 & 0.14 \\
\hline Quercetin & $>0.75$ & 0.375 & 1.25 & 0.625 \\
\hline Gallic acid & $>0.75$ & $>0.75$ & 0.75 & 0.75 \\
\hline Apigenin & 0.25 & 0.125 & 0.125 & 0.25 \\
\hline
\end{tabular}

${ }^{\text {a }}$ Isolated strain; ${ }^{\mathrm{b}}$ isolated strain; ${ }^{\mathrm{c}}$ ATCC $33862 ;{ }^{\mathrm{d}}$ ATCC 15442.

Table 7. Antibiofilm and anti-QS activity of tested compounds.

\begin{tabular}{|c|c|c|c|c|}
\hline Name & $\begin{array}{c}\text { Inhibition of } \\
\text { Bacterial Biofilm }\end{array}$ & $\begin{array}{c}\text { Inhibition of } \\
\text { Bacterial Biofilm }\end{array}$ & $\begin{array}{c}\text { Zone of } \\
\text { Inhibition * }\end{array}$ & $\begin{array}{l}\text { Violacein } \\
\text { Inhibition }\end{array}$ \\
\hline Juglone ${ }^{1}$ & $0 \%$ & $0 \%$ & 1 & $20 \%$ \\
\hline Caryophyllene-oxide $^{2}$ & $51 \%$ & $17 \%$ & 4 & $54 \%$ \\
\hline$\alpha$-Humulene ${ }^{3}$ & $58 \%$ & $56 \%$ & 5 & $40 \%$ \\
\hline Eugenol ${ }^{4}$ & $55 \%$ & $52 \%$ & 15 & $57.2 \%$ \\
\hline $\begin{array}{l}\text { 2-Phenylethyl- } \\
\text { isothiocyanate } 5\end{array}$ & $57 \%$ & $56 \%$ & 9 & $55.5 \%$ \\
\hline Caryophyllene ${ }^{6}$ & $0 \%$ & $0 \%$ & 5 & $50.8 \%$ \\
\hline Quercetin $^{7}$ & $0 \%$ & $52 \%$ & 0 & $0 \%$ \\
\hline Gallic acid $^{8}$ & $0 \%$ & $0 \%$ & 0 & $0 \%$ \\
\hline Apigenin ${ }^{9}$ & $48 \%$ & $58 \%$ & 0 & $0 \%$ \\
\hline
\end{tabular}

The major constituents of Juglans regia bark include quercitrin, quercetin and juglone (Table 5). The juglone showed significant inhibition (MIC range $0.005-0.075 \mathrm{mg} / \mathrm{mL}$ ) (Table 6); however, during antibiofilm and anti-QS sensing activities, no inhibition was recorded at the tested concentrations (Table 7). Quercetin showed a moderate inhibition of tested strains (MIC range $0.375-1.25 \mathrm{mg} / \mathrm{mL}$ ), and did not show any inhibition of the clinical strain S. aureus. Finally, although quercitrin showed very good docking results (Table S1), due to unavailability of this component, we were unable to test it. It was therefore concluded that components of Juglans regia bark may act synergistically towards the inhibition shown by the J. regia extract. 
a

$\mathrm{b}$

C

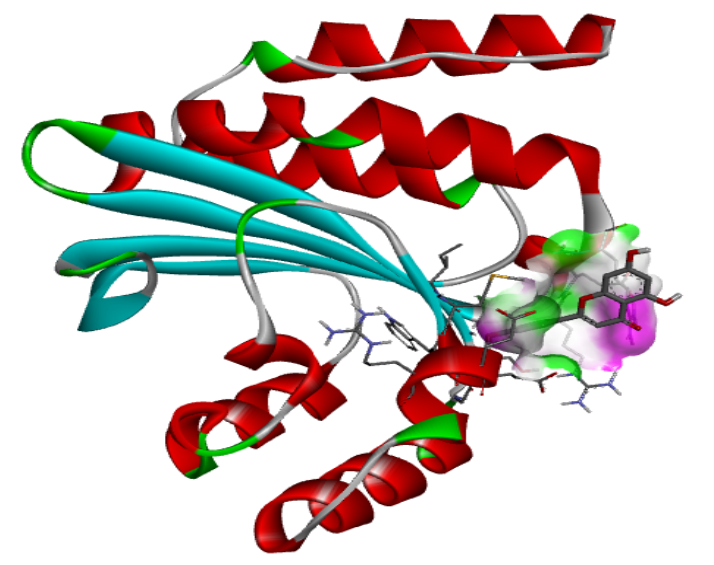

$\mathrm{d}$

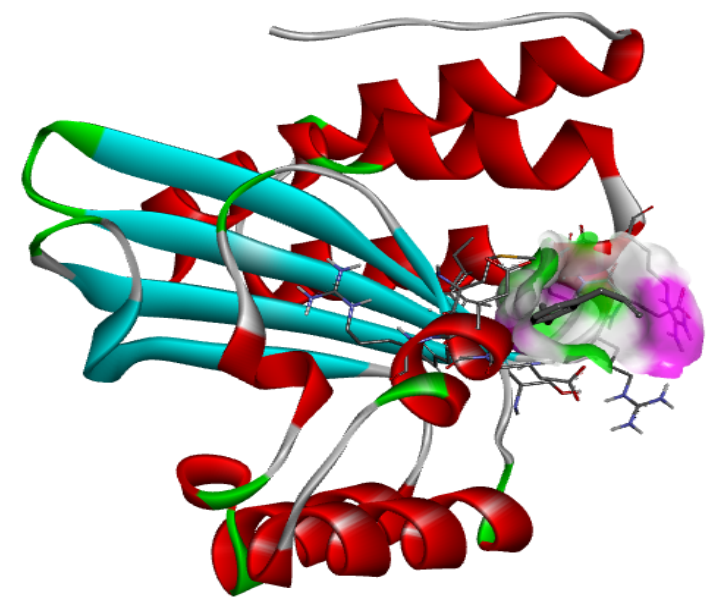

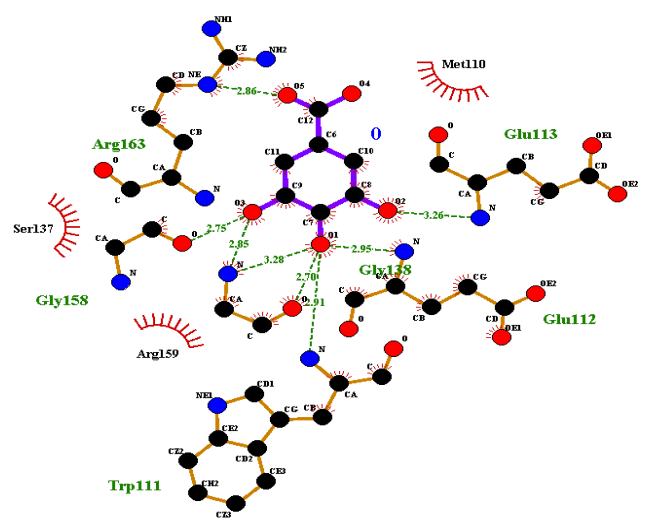
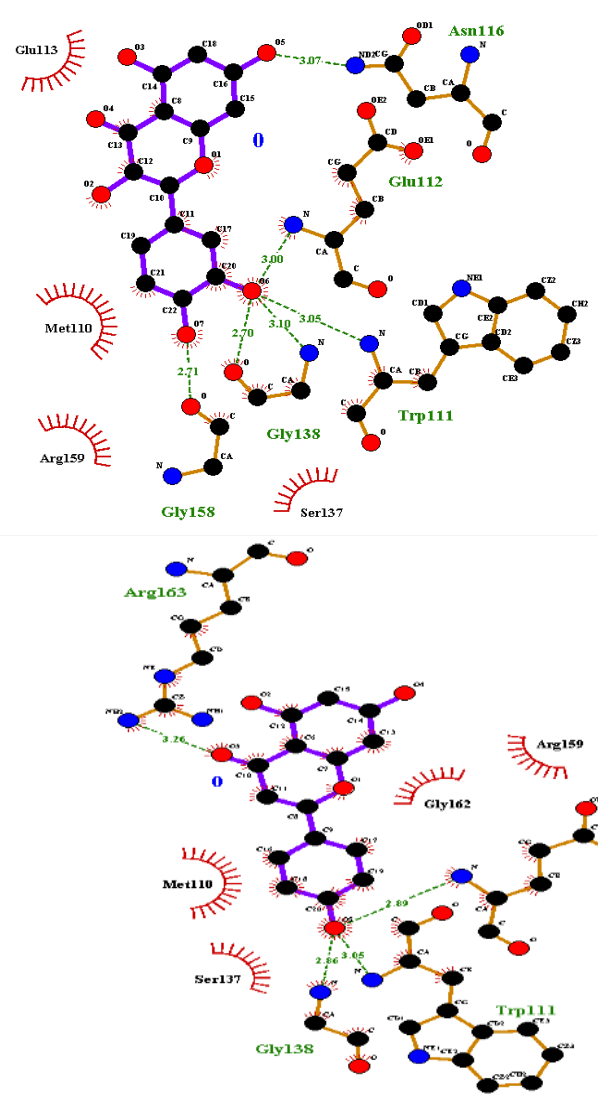

$\lambda_{7 \pi \Pi 1}^{\text {Angl63 }}$

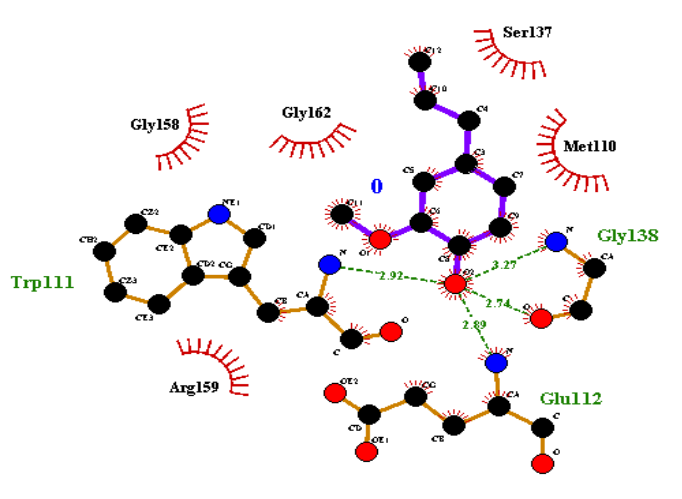

Figure 2. 3D interaction and $\mathrm{H}$, non-H bonding interactions of (a) gallic acid (pose 1), (b) quercitrin (pose 4), (c) apigenin (pose 3) and (d) eugenol (pose 6) inside binding sites of transcriptional regulator 3QP1 with pose rank 6. 
With regard to Eruca sativa oil, 2-phenylethylisothiocyanate was evaluated for antibacterial activity and inhibition was recorded (MIC range $0.005-0.18 \mathrm{mg} / \mathrm{mL}$ ) (Table 6). Interestingly, it showed a significant inhibition of biofilm formation (57\%) and anti-QS activities ( $9 \mathrm{~mm}$ zone of inhibition; $56 \%$ violacein inhibition) (Table 7 ).

The Punica granatum peel is a rich source of diverse polyphenols (Table 5), and strong antioxidant and antimicrobial properties have been reported. Additionally, during our preliminary investigations, peel extracts showed moderate antimicrobial activities (Table 3). The polyphenols including gallic acid, ellagic acid, quercetin and apigenin were tested for antimicrobial, antibiofilm and anti-QS properties. All tested compounds showed a moderate inhibition (MIC range $0.125-0.625 \mathrm{mg} / \mathrm{mL}$ ). Only apigenin inhibited all tested strains (Table 6). Contrarily, none of the compounds showed inhibition of quorum-sensing or antibiofilm properties at the tested concentrations (Table 7).

Likewise in Myristica fragrans, tested compounds included caryophyllene, caryophylleneoxide and $\alpha$-humulene, which were discussed above. Lastly, Azadirachta indica seed oil also showed significant antimicrobial (0.09-0.78\%), antibiofilm (58\%) and anti-QS-sensing activities (14 mm zone of inhibition; $52 \%$ violacein inhibition) as discussed earlier. However, the major components were not tested due to unavailability of these compounds.

\section{Conclusions}

It was concluded that Syzygium aromaticum, Juglans regia, Eruca sativa, Punica granatum and Azadirachta indica possessed antibacterial, antibiofilm and antiquorum-sensing properties. We therefore propose the use of these plant extracts or oils in formulations for the maintenance of oral hygiene as mentioned in ancient Ayurvedic literature.

Supplementary Materials: The following are available online at https: / www.mdpi.com/article / 10.3390/antibiotics10121504/s1: Figure S1: HPLC-DAD analysis of Punica granatum (peel extract). Figure S2: HPLC-DAD analysis of Juglon regia (bark extract). Figure S3: HPLC-DAD analysis of Juglon regia (root extract). Figure S4: HPLC-DAD analysis of Myristica fragrans (mace extract). Figure S5: HPLC-DAD analysis of Myristica fragrans (seed extract). Figure S6: HPLC-DAD-MSQToF analysis of Juglans regia (bark peel extract). Figure S7: HPLC-DAD-MS-QToF analysis of Juglans regia (root peel extract). Figure S8: HPLC-DAD-MS-QToF analysis of Myrictica fragrans (mace extract). Figure S9: HPLC-DAD-MS-QToF analysis of Myrictica fragrans (seed extract). Figure S10: HPLC-DAD-MS-QToF analysis of Punica granatum (peel extract). Figure S11: GC-MS chromatogram of Syzygium aromaticum oil. Figure S12: GC-MS chromatogram of Eruca sativa oil. Figure S13: GC-MS chromatogram of Azadirachta indica seed oil. Table S1: Primers sequencing parameters. Table S2: Optimized condition for polymerase chain reactions. Table S3: Optimized PCR conditions. Table S4: Identification of strains based on 16S rRNA gene sequence published in DNA database. Table S5: Identification of compounds from Juglans regia (bark peel extract) using HPLC-DAD-MSQToF. Table S6: Identification of compounds from Juglans regia (root peel extract) using HPLCDAD-MS-QToF. Table S7: Identification of compounds from Myrictica fragrans (mace extract) using HPLC-DAD-MS-QToF. Table S8: Identification of compounds from Myrictica fragrans (see extract) using HPLC-DAD-MS-QToF. Table S9: Identification of compounds from Punica granatum (peel extract) using HPLC-DAD-MS-QToF. Table S10: Docking score, $\mathrm{H}$ and non H-Bonding interactions of tested compounds.

Author Contributions: L.P., A.A. and U.H. presented basic concept of the project and methodology was written by A.A., U.H. and A.R. Software analysis was performed by A.R. and M.K. and validation of results was done by A.A., K.F. (Kainat Farooq), U.H., K.F. (Kenn Foubert) and L.p. The formal analysis, was performed by A.R., M.K. and K.F. (Kainat Farooq) and writing-original draft preparation was written by A.R., A.A., L.P., K.F. (Kenn Foubert) and U.H. did review and editing and the project was supervised by L.P., A.A., L.p. and A.A. worked for funding acquisition. All authors contributed to the article and approved the submitted version. All authors have read and agreed to the published version of the manuscript.

Funding: The Foundation "Plants for Health" is kindly acknowledged for financial support to Adnan Amin. 
Institutional Review Board Statement: The study was conducted according to the guidelines of the Declaration of Helsinki, and approved by the Ethical Review Board, Gomal University, D.I. Khan (2019).

Informed Consent Statement: Informed consent was obtained from all subjects involved in the study.

Conflicts of Interest: The authors declare no conflict of interest.

\section{References}

1. Kinane, D.F.; Stathopoulou, P.G.; Papapanou, P.N. Periodontal diseases. Nat. Rev. Dis. Primers 2017, 3, 17038. [CrossRef]

2. Schuster, G.S. Oral flora and pathogenic organisms. Oral Infect. 1999, 13, 757-774. [CrossRef]

3. Popova, C.; Dosseva-Panova, V.; Panov, V. Microbiology of Periodontal Diseases. A Review. Biotechnol. Biotechnol. Equip. 2013, 27, 3754-3759. [CrossRef]

4. Tang, B.; Gong, T.; Cui, Y.; Wang, L.; He, C.; Lu, M.; Chen, J.; Jing, M.; Zhang, A.; Li, Y. Characteristics of oral methicillin-resistant Staphylococcus epidermidis isolated from dental plaque. Int. J. Oral Sci. 2020, 12, 15. [CrossRef] [PubMed]

5. Donkor, E.S.; Kotey, F.C. Methicillin-Resistant Staphylococcus aureus in the Oral Cavity: Implications for Antibiotic Prophylaxis and Surveillance. Infect. Dis. 2020, 13, 1178633720976581. [CrossRef]

6. Naranjo, A.A.; Triviño, M.L.; Jaramillo, A.; Betancourth, M.; Botero, J.E. Changes in the subgingival microbiota and periodontal parameters before and 3 months after bracket placement. Am. J. Orthod. Dentofac. Orthop. 2006, 130, 275.e17-275.e22. [CrossRef]

7. Murshid, E.Z. Diet, oral hygiene practices and dental health in autistic children in Riyadh, Saudi Arabia. Oral Health Dent. Manag. 2014, 13, 91-96.

8. Bradshaw, D.J.; Marsh, P.D. Analysis of pH-driven disruption of oral microbial communities in vitro. Caries Res. 1998, 32, 456-462. [CrossRef] [PubMed]

9. Lalla, E.; Cheng, B.; Lal, S.; Kaplan, S.; Softness, B.; Greenberg, S.E.; Goland, R.S.; Lamster, I.B. Diabetes mellitus promotes periodontal destruction in children. J. Clin. Periodont. 2007, 34, 294-298. [CrossRef]

10. Kim, J.; Amar, S. Periodontal disease and systemic conditions: A bidirectional relationship. Odontology 2006, 94, 10-21. [CrossRef] [PubMed]

11. Kim, Y.G.; Lee, H.J.; Gwon, G.; Kim, S.; Park, J.G.; Lee, J. Essential Oils and Eugenols Inhibit Biofilm Formation and the Virulence of Escherichia coli O157:H7. Sci. Rep. 2016, 6, 36377. [CrossRef] [PubMed]

12. Heller, D.; Helmerhorst, E.; Oppenheim, F. Saliva and Serum Protein Exchange at the Tooth Enamel Surface. J. Dent. Res. 2016, 96, 437-443. [CrossRef]

13. Weerkamp, A.H.; Uyen, H.M.; Busscher, H.J. Effect of zeta potential and surface energy on bacterial adhesion to uncoated and salivacoated human enamel and dentin. J. Dent. Res. 1988, 67, 1483-1487. [CrossRef] [PubMed]

14. Jones, A. Ultrastructure of Proteus mirabilis swarmer cell rafts and role of swarming in catheter associated urinary tract infection. Infect. Immun. 2014, 72, 3941-3950. [CrossRef]

15. Waters, C.M.; Bassler, B.L. Quorum Sensing: Cell-to-Cell Communication in Bacteria. Annu. Rev. Cell Dev. Biol. 2005, 21, 319-346. [CrossRef]

16. Gibot, A.S. Fighting the enemy properly. Critical. Care. Med. 2004, 32, 1223-1224. [CrossRef] [PubMed]

17. Musthafa, K.S.; Ravi, A.V.; Annapoorani, A.; Packiavathy, S.V.; Pandian, S.K. Evaluation of Anti-Quorum-Sensing Activity of Edible Plants and Fruits through Inhibition of the N-Acyl-Homoserine Lactone System in Chromobacterium violaceum and Pseudomonas aeruginosa. Chemotherapy 2010, 56, 333-339. [CrossRef]

18. Brackman, G.; Defoirdt, T.; Miyamoto, C.; Bossier, P.; Van Calenbergh, S.; Nelis, H.; Coenye, T. Cinnamaldehyde and cinnamaldehyde derivatives reduce virulence in Vibrio spp. by decreasing the DNA-binding activity of the quorum sensing response regulator LuxR. BMC Microbiol. 2008, 8, 149. [CrossRef]

19. Naseem, S.; Hashmi, K.; Fasih, F.; Sharafat, S.; Khanani, R. In vitro evaluation of antimicrobial effect of miswak against common oral pathogens. Pak. J. Med. Sci. 2014, 30, 398-403. [CrossRef]

20. Almas, K. The antimicrobial effects of seven different types of Asian chewing sticks. Odonto-Stomatol. Trop. 2001, 24, 17-20.

21. Al Lafi, T.; Ababneh, H. The effect of the extract of the miswak (chewing sticks) used in Jordan and the Middle East on oral bacteria. Int. Dent. J. 1995, 45, 218-222. [PubMed]

22. Marwat, S.K.; Khan, M.A.; Ahmad, M.; Zafar, M.; Rehman, F.U. Ethanophytomedicine for the treatment of various Diseases in D.I.Khan District. Sarhad. J. Agric. 2008, 24, 33-39.

23. Carrol, D.H.; Chassagne, F.; Dettweiler, M.; Quave, C.L. Antibacterial activity of plant species used for oral health against Porphyromonas gingivalis. PLoS ONE 2020, 15, e0239316. [CrossRef]

24. Lewis, W.; Elvin-Lewis, M. Oral hygiene. In Medical Botany, 2nd ed.; John Wiley \& Sons: Hoboken, NJ, USA, 2003.

25. Niazi, F.; Naseem, M.; Khurshid, Z.; Zafar, M.; Almas, K. Role of Salvadora persica chewing stick (miswak): A natural toothbrush for holistic oral health. Eur. J. Dent. 2016, 10, 301-308. [CrossRef] [PubMed]

26. Lakshmi, T.; Krishnan, V.; Rajendran, R.; Madhusudhanan, N. Azadirachta indica: A herbal panacea in dentistry-An update. Pharmacogn. Rev. 2015, 9, 41-44. [CrossRef]

27. Nagesh, L.; Amith, H. Effect of Oil Pulling on Plaque and Gingivitis. JOHCD 2007, 1, 12-18. [CrossRef] 
28. El-Aziz, G.S.A.; El-Fark, M.O.; Hamdy, R.M. Protective effect of Eruca sativa seed oil against oral nicotine induced testicular damage in rats. Tissue Cell 2016, 48, 340-348. [CrossRef]

29. Gao, Q.; Li, X.; Huang, H.; Guan, Y.; Mi, Q.; Yao, J. The Efficacy of a Chewing Gum Containing Phyllanthus emblica Fruit Extract in Improving Oral Health. Curr. Microbiol. 2018, 75, 604-610. [CrossRef]

30. Kaliamoorthy, S.; Pzahani, A.; Nagarajan, M.; Mayyappan, A.; Rayar, S. Comaparing the effect of coconut oil pulling practice with oil pulling using sesame oil in plaque-induced gingivitis: A prospective comparative interventional study. J. Nat. Sc. Biol. Med. 2018, 9, 165-168. [CrossRef]

31. Kumar, V.; Shanbhag, L. Oil pulling for maintaining oral hygiene-A review. J. Trad. Complement. Med. 2017, 7, 106-109.

32. Katsure, H.; Tsuliyama, R.; Suzuki, A.; Kobayashi, M. In Vitro Antimicrobial Activities of Bakuchiol against Oral Microorganisms. Amtimicrob. Agent. Chemother. 2001, 45, 3009-3013. [CrossRef]

33. Chaieb, K.; Hajlaoui, H.; Zmantar, T.; Ben Kahla-Nakbi, A.; Rouabhia, M.; Mahdouani, K.; Bakhrouf, A. The chemical composition and biological activity of clove essential oil, Eugenia caryophyllata (Syzigium aromaticum L. Myrtaceae): A short review. Phytother. Res. 2007, 21, 501-506. [CrossRef]

34. Kimura, S.S.; Tung, Y.C.; Pan, M.H.; Weisu, N.; Lai, N.W.; Cheng, K. CBlack garlic: A critical review of its production, bioactivity, and application. J. Food. Drug Anal. 2017, 25, 62-70. [CrossRef]

35. Rao, H.A.; Bhat, S.S.; Hegde, S.; Jhamb, V. Efficacy of garlic extract and chlorhexidine mouthwash in reduction of oral salivary microorganisms, an in vitro study. Anc. Sci. Life 2014, 34, 85-88. [CrossRef]

36. Jalayer-Naderi, N.; Niakan, E.; Mohamadi-Motlagh, M. The antibacterial activity of methanolic Anacyclus pyrethrum and Pistacia lentiscus L. extract on Escherichia coli. Iran. J. Microbiol. 2016, 8, 372-376.

37. Sharma, M.; Tandon, S.; Nayak, U.A.; Kappadi, D.; Rathore, A.S.; Goyal, A. Calotropis gigantea extract as a potential anticariogenic agents against Streptococcus mutans: An in vivo comparative evaluation. J. Conserv. Dent. 2017, 20, 174-179. [CrossRef] [PubMed]

38. Gohil, K.; Patel, J.; Gajjar, A. Pharmacological review on Centella asiatica: A potential herbal cure-all. Indian J. Pharm. Sci. 2010, 72, 546-556. [CrossRef]

39. D'Souza, S.P.; Chavannavar, S.V.; Kanchanashri, B.; Niveditha, S.B. Pharmaceutical Perspectives of Spices and Condiments as Alternative Antimicrobial Remedy. J. Evid. Based Integr. Med. 2017, 22, 1002-1010. [CrossRef]

40. Shafiei, Z.; Shuhairi, N.N.; Yap, N.M.F.S.; Sibungkil, C.-A.H.; Latip, J. Antibacterial Activity of Myristica fragransagainst Oral Pathogens. Evid. Based Complement. Altern. Med. 2012, 2012, 825362. [CrossRef] [PubMed]

41. Prasad, D.; Kunnaiah, R. Punica granatum: A review on its potential role in treating periodontal disease. J. Indian Soc. Periodontol. 2014, 18, 428. [CrossRef]

42. Amalraj, A.; Gopi, S. Medicinal properties of Terminalia arjuna (Roxb.) Wight \& Arn.: A review. J. Tradit. Complement. Med. 2016, 7 , 65-78. [CrossRef]

43. Kregiel, D.; Pawlikowska, E.; Antolak, H. Urtica spp.: Ordinary Plants with Extraordinary Properties. Molecules 2018, $23,1664$. [CrossRef]

44. Pardeep, K.R. Ethno medicinal plants used for oral health care in India. Int. J. Herb. Med. 2014, 2, 81-87.

45. Cariri, M.; Givisiez, P.E.N.; Sousa, F.G.; Magnani, M.; Souza, E.L.; Spricigo, D.A.S. Biofilm-forming and antimicrobial resistance traits of staphylococci from goat dairy plants. J. Infect. Dev. Ctries. 2016, 10, 932-938.

46. Amin, A.; Hanif, M.; Abbas, K.; Raman, M.; Rasheed, A.; Zaman, A.; Pieters, L. Studies on effects of umbelliferon derivatives against periodontal bacteria; antibiofilm, inhibition of quorum sensing and molecular docking analysis. Microb. Pathog. 2020, 144, 104184. [CrossRef]

47. Pitcher, D.G.; Saunders, N.A.; Owen, R.J. Rapid extraction of bacterial genomic DNA with guanidium thiocyanate. Lett. Appl. Microbiol. 1989, 8, 151-156. [CrossRef]

48. Joshi, M.; Deshpande, J.D. Polymerase chain reaction: Methods, principles an application. Int. J. Biomed. Res. 2010, 2, 81-97. [CrossRef]

49. Yu, S.; Jensen, V.; Seeliger, J.; Feldmann, I.; Weber, S.; Schleicher, E.; Häussler, S.; Blankenfeldt, W. Structure Elucidation and Preliminary Assessment of Hydrolase Activity of PqsE, the Pseudomonas Quinolone Signal (PQS) Response Protein. Biochemistry 2009, 48, 10298-10307. [CrossRef] [PubMed]

50. Chen, G.; Swem, L.R.; Swem, D.L.; Stauff, D.L.; O’Loughlin, C.T.; Jeffrey, P.D.; Bassler, B.; Hughson, F.M. A Strategy for Antagonizing Quorum Sensing. Mol. Cell 2011, 42, 199-209. [CrossRef]

51. Discovery Studio Visualizer, version 2.0.; Accelrys Software Inc.: San Diego, CA, USA, 2012.

52. Trott, O.; Olson, A.J. AutoDock Vina: Improving the speed and accuracy of docking with a new scoring function, efficient optimization, and multithreading. J. Comput. Chem. 2010, 31, 455-461. [CrossRef]

53. Weseler, A.H.K.; Geiss, E.; Saller, R.; Retching, J. A novel colorimetric broth microdilution method to determine the minimum inhibitory concentration (MIC) of antibiotics and essential oils against Helicobacter pylori. Pharmazie 2005, 60, 498-502. [PubMed]

54. Bazargani, M.M.; Rohloff, J. Antibiofilm activity of essential oils and plant extracts against Staphylococcus aureus and Escherichia coli biofilms. Food Control. 2016, 61, 156-164. [CrossRef]

55. Koh, K.H.; Tham, F.-Y. Screening of traditional Chinese medicinal plants for quorum-sensing inhibitors activity. J. Microbiol. Immunol. Infect. 2011, 44, 144-148. [CrossRef]

56. McLean, R.J.; Pierson, L.S.; Fuqua, C. A simple screening protocol for the identification of quorum signal antagonists. J. Microbiol. Methods 2004, 58, 351-360. [CrossRef] [PubMed] 
57. Raafa, K. Phytochemical analysis of Juglans regia oil and kernel exploring their antinociceptive and anti-inflammatory potentials utilizing combined bio-guided GC-FID, GC-MS and HPLC analyses. Rev. Brasil. Farmacogn. 2018, 28, 358-368. [CrossRef]

58. Ho, K.-V.; Lei, Z.; Sumner, L.W.; Coggeshall, M.V.; Hsieh, H.-Y.; Stewart, G.C.; Lin, C.-H. Identifying Antibacterial Compounds in Black Walnuts (Juglans nigra) Using a Metabolomics Approach. Metabolites 2018, 8, 58. [CrossRef] [PubMed]

59. Singh, B.; Singh, J.P.; Kaur, A.; Singh, N. Phenolic compounds as beneficial phytochemicals in pomegranate (Punica granatum L.) peel: A review. Food Chem. 2018, 261, 75-86. [CrossRef] [PubMed]

60. Melvita, A.; Ju, Y.H. Separation of azadirachtin and other limonoids from crude neem oil via solvent precipitation. Sepr. Purif. Technol. 2010, 74, 219-224. [CrossRef]

61. Khoobchandani, M.; Ganesh, N.; Gabbanini, S.; Valgimigli, L.; Srivastava, M. Phytochemical potential of Eruca sativa for inhibition of melanoma tumor growth. Fitoterapia 2011, 82, 647-653. [CrossRef] [PubMed]

62. Cu, J.-Q.; Perineau, F.; Goepfert, G. GC/MS Analysis of Star Anise Oil. J. Essent. Oil Res. 1990, 2, 91-92. [CrossRef]

63. Luis, A.; Sousa, S.; Wackerlig, A.; Dobusch, D.; Daurte, A.P.; Pereira, L.; Domingues, L. Star anise (Illicium verum Hook. f.) essential oil: Antioxidant properties and antibacterial activity against Acinetobacter baumannii. Flavor Frag. J. 2019, 34, 260-270. [CrossRef]

64. Kwiatkowski, P.; Pruss, A.; Masiuk, H.; Mnichowska-Polanowska, M.; Kaczmarek, M.; Giedrys-Kalemba, S.; Dołegowska, B.; Zielińska-Bliźniewska, H.; Olszewski, J.; Sienkiewicz, M. The effect of fennel essential oil and trans-anethole on antibacterial activity of mupirocin against Staphylococcus aureus isolated from asymptomatic carriers. Postepy Dermatol. Allergol. 2019, 36, 308-314. [CrossRef] [PubMed]

65. Rajaa, S.I.; Ahmada, S.I.; Irshadadb, M.; Wanid, W.A.; Siddiqia, W.A.; Shreaz, S. Anticandidal activity of ethanolic root extract of Juglans regia (L.): Effecton growth, cell morphology, and key virulence factors. J. Mycol. Méd. 2017, 27, 476-486. [CrossRef] [PubMed]

66. Matulyte, I.; Marksa, M.; Ivanauskas, L.; Kalveniene,, Z.; Lazauskas, R.; Bernatoniene, J. GC-MS Analysis of the Composition of the Extracts and Essential Oil from Myristica fragrans Seeds Using Magnesium Aluminometasilicate as Excipient. Molecules 2019, 24, 1062. [CrossRef]

67. Gupta, A.D.; Bansal, V.K.; Babu, V.; Maithil, N. Chemistry, antioxidant and antimicrobial potential of nutmeg (Myristica fragrans Houtt). J. Genet. Eng. Biotechnol. 2013, 11, 25-31. [CrossRef]

68. Shafiq, M.I.; Ahmad, M.; Rasul, A.; Samra, Z.Q.; Qadir, M.A.; Mazhar, S.; Ali, A. Chemical Composition of the Essential Oils of Nutmeg and Mace by GC-FID/MS Indigenous to Pakistan and Evaluation of their Biological Activities. Lat. Am. J. Pharm. 2016, 35, 2176-2184.

69. Alma, M.H.; Ertaş, M.; Nitz, S.; Kollmannsberger, H. Chemical composition and content of essential oil from the bud of cultivated Turkish clove (Syzygium aromaticum L.). BioResources 2007, 2, 265-269. [CrossRef]

70. Zhao, X.; Yuan, Z.; Faung, Y.; Yin, Y.; Feng, L. Charcaterization and evaluation of major anthocyanins in pomegranate (Punica granatum L.) peel of different cultivars and their development phases. Eur. Food. Res. Technol. 2013, 236, 109-117. [CrossRef]

71. Venkataraghavan, K.; Shah, S.; Choudhary, P.; Mohammad, S.; Trivedi, K.; Shah, S.G. Evaluation of antimicrobial effect of azadirachtin plant extract (Soluneem ${ }^{\mathrm{TM}}$ ) on commonly found root canal pathogenic microorganisms (viz. Enterococcus faecalis) in primary teeth: A microbiological study. J. Indian Soc. Pedod. Prev. Dent. 2016, 34, 210-216. [CrossRef] 